\title{
Sociodemographic, personal, peer, and familial predictors of e-cigarette ever use in ESPAD Ireland: A forward stepwise logistic regression model
}

\author{
Joan Hanafin ${ }^{1,2}$, Salome Sunday ${ }^{1,2}$, Luke Clancy ${ }^{1,2}$
}

\begin{abstract}
INTRODUCTION E-cigarette ever use has risen significantly in recent years in Ireland, similar to trends elsewhere in Europe, the United States, and Asia-Pacific region. Results from ESPAD Ireland (European School Survey Project on Alcohol and other Drugs) show teenage e-cigarette ever use increased from 18\% (2015) to $37 \%$ (2019). Given this increase, our aim is to profile e-cigarette ever users and never users in this age group; to examine sociodemographic, personal, peer, and familial factors associated with e-cigarette ever use; and to suggest appropriate measures to reduce use.

METHODS A nationally representative stratified random sample of 50 ESPAD schools was surveyed in 2019, with 3495 students aged 15-17 years. Bivariate and multivariable logistic regression analyses were performed using Stata version 16. RESULTS E-cigarette ever use was significantly associated with ever smoking (AOR $=4.15 ; 95 \%$ CI: $1.29-13.41)$, ever cannabis use (AOR=2.21; 95\% CI: $1.11-4.41)$ and ever inhalants use (AOR=2.51; 95\% CI: 1.07-5.88). Children of university-educated mothers had significantly higher odds of e-cigarette ever use $(\mathrm{AOR}=3.46$; 95\% CI: 1.40-8.54). Associated with reduced AORs were reading books for enjoyment ( $\mathrm{AOR}=0.32$; 95\% CI: 0.16-0.64), living in households where smoking was regulated ( $\mathrm{AOR}=0.53 ; 95 \% \mathrm{CI}$ : 0.30-0.94), and perceiving moderate risk in trying e-cigarettes once or twice $(\mathrm{AOR}=0.20 ; 95 \% \mathrm{CI}: 0.07-0.67)$.

CONCLUSIONS E-cigarette ever use is part of a pattern of teenage polysubstance use including cigarette smoking, providing some support for the common liability theory. Regulation of smoking in the home, reading for enjoyment, and perceiving risk from e-cigarette use are associated with decreased likelihood of ever use, and higher parental education with increased likelihood. Thus, health education emphasizing the role of parents and risks of e-cigarette use is indicated to reduce the rise in e-cigarette ever use in teenagers.
\end{abstract}

\section{AFFILIATION \\ 1 TobaccoFree Research Institute Ireland, Dublin, Ireland \\ 2 Technological University Dublin, Dublin, Ireland \\ CORRESPONDENCE TO Luke Clancy. TobaccoFree Research Institute Ireland, Dublin, Ireland. E-mail: Iclancy@tri.ie ORCID ID: https://orcid.org/ 0000-0003-2407-2263 \\ KEYWORDS \\ parental education, e-cigarette ever use, smoking regulation, polysubstance use, teenagers}

Received: 2 July 2021 Revised: 24 November 2021 Accepted: 24 November 2021

\section{INTRODUCTION}

E-cigarette use among teenagers in Ireland has risen significantly in recent years and findings from ESPAD Ireland (European School Survey Project on Alcohol and Other Drugs) show that in 2019 in Ireland, among children born in 2003, e-cigarette ever use prevalence was $37 \%{ }^{1}$. Increasing prevalence of ever use of e-cigarettes in Ireland reflects similar trends elsewhere in Europe ${ }^{2}$, the United States (US) $)^{3,4}$ and the Asia-Pacific region ${ }^{5}$. In Ireland, current use (30-day) of e-cigarettes among adolescents has also increased significantly from $10 \%$ in 2015 to $18 \%$ in $2019^{6}$ and has been linked to significantly increased smoking prevalence among teenage boys ${ }^{7}$.

The 2019 ESPAD survey ${ }^{2}$ of 99647 students from 35 countries in Europe reported an average of $40 \%$ 
ever use of e-cigarettes among students aged 16 years, ranging from $18 \%$ in Serbia to $65 \%$ in Lithuania. The United Kingdom did not participate in ESPAD in 2019 but prevalence of ever use of e-cigarettes among Scottish 15-year-olds was reported at 35\% in 2018, with boys more likely than girls to have ever used e-cigarettes ${ }^{8}$. Increasing prevalence is not reported everywhere, however. In England, reported prevalence for ever use of e-cigarettes among school pupils aged $11-15$ years was $25 \%$ in 2018 , the same as in $2016^{9}$. E-cigarettes are now the most commonly used tobacco product among young people in the $\mathrm{US}^{10}$.

A narrative review ${ }^{11}$ of e-cigarette prevalence in Europe among adults and young people found that ever use ranged from $5.5 \%$ to $56.6 \%$. Current smokers of conventional cigarettes showed the highest prevalence for e-cigarette ever use with $20.4 \%$ to $83.1 \%$, followed by ex-smokers with $7 \%$ to $15 \%$. In European countries, there is a higher prevalence of e-cigarette use among males, adolescents and young adults, smokers of conventional cigarettes, and former smokers.

There are concerns that the growing popularity of e-cigarettes promotes tobacco experimentation, particularly among younger children ${ }^{12}$. A Welsh study of primary schoolchildren found that children as young as 7 years have general awareness of e-cigarettes. They perceived vaping to be healthier than smoking, had some recognition that e-cigarettes were used for smoking cessation but showed limited understanding of any health harms ${ }^{12}$.

The longitudinal analysis of Tokle ${ }^{13}$ found 'a systematic pattern in which adolescents account for vaping as a time-limited trend'. Within this Norwegian sample, over a four-year period, e-cigarettes were devalued from novelty and transgression to childish and uninteresting, leading to the conclusion that e-cigarettes represented fashionable experimentation rather than steady user patterns. Among adolescent cigarette experimenters, using e-cigarettes has been found to be positively and independently associated with progression to current established smoking, suggesting that e-cigarettes 'do not divert from, and may encourage, cigarette smoking in this population'14.

In Ireland, ESPAD 2019 findings show that, among children born in 2003 who ever used e-cigarettes, $67 \%$ have never smoked cigarettes ${ }^{1}$, representing a worrying new trend of initiation into nicotine addiction. From 1995 to 2015, smoking prevalence has been decreasing in Ireland, markedly so, among Irish teenagers ${ }^{15}$. Now, however, for the first time in 25 years this decrease has stalled, with prevalence rates (30-day use) among people aged 15-17 years in 2019 remaining the same $(14.4 \%)$ as they were in 2015 , accounted for by an increase in smoking prevalence among boys (16.2\%), and a decrease among girls $(12.8 \%)^{1}$. This halt in smoking prevalence reduction has been accompanied by a rising prevalence of e-cigarette use, particularly among boys, pointing to a possible link ${ }^{6,7}$.

The association between cigarette and e-cigarette use in teenagers is established but the mechanisms are uncertain. The longest-standing theory is the Gateway Theory ${ }^{16}$ which concerns the centrality of nicotine addiction in the progression to other drugs, but it is insufficient to explain fully the progression to cigarettes from e-cigarettes. The Common Liability Theory ${ }^{17}$ allows for wider inputs from environmental and genetic influences giving rise to the use of various tobacco products and psychoactive substances ${ }^{16-20}$, while the Catalyst Model ${ }^{18}$ helps consider the factors influencing initiation and progression, which could possibly extend to a diversion model preventing progression to smoking ${ }^{19}$. Although marketed as a smoking cessation tool, e-cigarettes are rarely used for this purpose in youth ${ }^{21}$. Among adolescents in Ireland, the main motivation for using e-cigarettes was curiosity (66\%) and because friends offered ( $29 \%$ ), while only $3.4 \%$ said that their motivation for beginning to use e-cigarettes was for smoking cessation ${ }^{1}$.

E-cigarette ever use has increased among people aged 15-17 years in Ireland from 18\% to 37\% between 2015 and $2019^{1,22}$. Our aim in this study is to profile e-cigarette ever users and never users in this age group; to examine associations with e-cigarette use; and to suggest appropriate measures to reduce use. In our model, we include sociodemographic, personal, peer, and familial associations which are available in the ESPAD dataset and known to be associated with teenage substance use.

\section{METHODS}

\section{Design, sample, data collection}

The ESPAD survey is the largest quadrennial cross- 
national project on adolescent substance use in the world, having the overall aim of repeatedly collecting comparable data on substance use among young people in some 35 European countries (www.espad. org). In the 2019 Ireland (Republic) arm of the ESPAD survey, a total of 3565 students aged 15, 16 and 17 years from a nationally representative, stratified random sample of 50 schools were surveyed. Data were collected between March and May 2019 and full accounts of the sampling, data collection and cleaning procedures have been reported elsewhere ${ }^{2}$. Following data cleaning, the final sample comprised 3495 students.

\section{Dependent variable}

Prevalence of e-cigarette ever use was measured by the question: 'Have you ever used e-cigarettes?', No; Yes, more than 12 months ago; Yes, in the last 12 months; and Yes, in the last 30 days; recoded as ever use, no versus yes.

\section{Independent variables}

Sociodemographic, personal, peer and familial characteristics are shown in detail with full question and answer categories in the Supplementary file and we summarize them here.

Sociodemographic variables included age, sex, parental education level, perceived family wealth, and household composition. Variables measuring personal risk behaviors as well as potentially protective behaviors were: use of cigarettes, alcohol, cannabis (including problem cannabis use (CAST [Cannabis Abuse Screening Test], a 6-item, 5-point scale, Cronbach's alpha 0.83), inhalants, tranquilizers (with prescription); age of smoking and alcohol initiation; problems with social media use (3-item, 5-point Likert scale, Cronbach's alpha 0.67), internet use (14-item, 5-point Likert scale, Cronbach's alpha 0.92), online gaming (12-item, 5-point Likert scale, Cronbach's alpha 0.95), and gambling; missing school due to truancy; reading books other than school books, actively participating in sport, having other hobbies, and average school grade. Variables measuring peer risk activities were: how many of their friends smoke, drink alcohol, get drunk, use cannabis, tranquilizers/ sedatives, ecstasy, and inhalants. The variable 'peer support' measured friends' help, support, sharing and communication (4-item, 7-point Likert scale,
Cronbach's alpha 0.94). Familial variables measured familial support (a 4-item, 7-point Likert scale, Cronbach's alpha 0.92), relationship with parents, and household rules about smoking (smoking regulation).

\section{Statistical analysis}

Pearson's $\chi^{2}$ test (for categorical variables) and Student's t-test (for continuous variables) were conducted to compare differences in e-cigarette use between ever users and never users in relation to the independent variables above (Table 1). All variables in the study were adjusted by the dependent variable (e-cigarette ever use) using Spearman's correlation coefficient and variance inflation factor (VIF) as appropriate between variables, and a VIF $<5$ was used to detect multicollinearity. A univariate logistic regression analysis was then performed to assess the association of e-cigarette ever use with the sociodemographic, personal, peer and familial characteristics of respondents. The results are presented in Table 2 as crude odds ratios (ORs) and their respective $95 \%$ confidence intervals (95\% CIs). This was followed by a stepwise logistic regression analysis to assess predictors of e-cigarette ever use after entering all the variables in the model, and only variables with a $\mathrm{p}<0.7$ were retained in the final model (Table 2). Adjusted odds ratios (AORs) and their 95\% confidence intervals were estimated and associations with a $\mathrm{p}<0.05$ were considered statistically significant. All analyses were performed using Stata version 16 .

\section{RESULTS}

\section{Characteristics of e-cigarette ever users, bivariate analyses}

A total of 3495 students were included in the analysis. Sample characteristics of e-cigarette ever use among people aged 15-17 years are shown in Table 1 . Overall, $36.6 \%(n=1278)$ of students in the sample had ever used e-cigarettes. Girls were more likely than boys to be never users $(65.6 \%, n=1200)$. In these bivariate analyses, there were significant differences between e-cigarette ever users and never users according to: sex, parental education level, household composition, absenteeism, ever and current smoking, skipping school; ever, current, and binge alcohol use; ever, current, cannabis use and problem cannabis use; ever use of tranquilizers with or without prescription; perceived risks of smoking e-cigarettes; and familial 
Table 1. Sample characteristics of e-cigarette ever users and never users aged 15-17 years ( $N=3495)$

\begin{tabular}{|c|c|c|c|c|}
\hline Characteristics & $\begin{array}{l}\text { Total } \\
n(\%)\end{array}$ & $\begin{array}{c}\text { E-cigarette never } \\
\text { users } \\
n(\%)\end{array}$ & $\begin{array}{c}\text { E-cigarette ever } \\
\text { users } \\
n(\%)\end{array}$ & $p^{*}$ \\
\hline Total & $3495(100)$ & $2209(63.4)$ & $1278(36.6)$ & \\
\hline Sex & & & & 0.004 \\
\hline Female & $1835(52.5)$ & $1200(65.6)$ & $630(34.4)$ & \\
\hline Male & $1660(47.5)$ & $1009(60.9)$ & $648(39.1)$ & \\
\hline Age (years) & & & & 0.263 \\
\hline 15 & $796(22.8)$ & $523(65.7)$ & $272(34.2)$ & \\
\hline 16 & 1949 (55.7) & $1219(62.7)$ & $724(37.3)$ & \\
\hline 17 & $750(21.5)$ & $467(62.3)$ & $282(37.7)$ & \\
\hline Father's education level & & & & 0.012 \\
\hline Some secondary school or completed primary school & $723(24.4)$ & $423(58.5)$ & $300(41.5)$ & \\
\hline Completed secondary school & $608(20.6)$ & $379(62.3)$ & $229(37.7)$ & \\
\hline College or university & $1628(55.0)$ & $1057(64.9)$ & $571(35.1)$ & \\
\hline Mother's education level & & & & $<0.001$ \\
\hline Some secondary school or completed primary school & $364(11.8)$ & $245(67.3)$ & $119(32.7)$ & \\
\hline Completed secondary school & $681(22.0)$ & $494(72.5)$ & $187(27.5)$ & \\
\hline College or university & $2046(66.2)$ & $1236(60.4)$ & $810(39.6)$ & \\
\hline Perceived wealth & & & & 0.217 \\
\hline About the same & $1473(44.0)$ & $951(64.6)$ & $522(35.4)$ & \\
\hline Much better off & $523(15.6)$ & $322(61.6)$ & $201(38.4)$ & \\
\hline Better off & 1032 (30.8) & $664(64.3)$ & $368(35.7)$ & \\
\hline Less well off & $321(9.6)$ & $190(59.2)$ & $131(40.8)$ & \\
\hline Household composition & & & & 0.499 \\
\hline Two parents & $2602(78.4)$ & $1661(63.8)$ & $941(36.2)$ & \\
\hline One parent & $524(15.8)$ & $321(61.3)$ & $203(38.7)$ & \\
\hline Blended families & $195(5.9)$ & $121(62.0)$ & $74(38.0)$ & \\
\hline Average grade & & & & 0.148 \\
\hline$A$ and $B$ & $1511(45.7)$ & $990(65.5)$ & $521(34.5)$ & \\
\hline $\mathrm{C}$ & 1389 (42.0) & $862(62.2)$ & $524(37.8)$ & \\
\hline D or lower & $407(12.3)$ & $254(62.4)$ & $153(37.6)$ & \\
\hline Read books & & & & $<0.001$ \\
\hline No & $2636(76.9)$ & $1560(59.2)$ & $1076(40.8)$ & \\
\hline Yes & $794(23.1)$ & $613(77.2)$ & $181(22.8)$ & \\
\hline Actively participate in sports & & & & 0.906 \\
\hline No & 551 (15.9) & $350(63.5)$ & $201(36.5)$ & \\
\hline Yes & 2904 (84.1) & $1837(63.3)$ & 1067 (36.7) & \\
\hline Other hobbies ${ }^{\mathrm{a}}$ & & & & $<0.001$ \\
\hline No & $1484(44.5)$ & 889 (59.9) & $595(40.1)$ & \\
\hline Yes & 1854 (55.5) & $1229(66.3)$ & $625(33.71)$ & \\
\hline Age of alcohol initiation (years) & & & & $<0.001$ \\
\hline$<13$ & $844(35.8)$ & $333(39.5)$ & $511(60.5)$ & \\
\hline$\geq 14$ & $1514(64.2)$ & $870(57.5)$ & $644(42.5)$ & \\
\hline
\end{tabular}


Table 1. Continued

\begin{tabular}{|c|c|c|c|c|}
\hline Characteristics & $\begin{array}{l}\text { Total } \\
n(\%)\end{array}$ & $\begin{array}{c}\text { E-cigarette never } \\
\text { users } \\
n(\%)\end{array}$ & $\begin{array}{c}\text { E-cigarette ever } \\
\text { users } \\
n(\%)\end{array}$ & $p *$ \\
\hline Age of smoking initiation (years) & & & & 0.001 \\
\hline$<13$ & $374(33.7)$ & $62(16.6)$ & $312(83.4)$ & \\
\hline$\geq 14$ & $736(66.3)$ & $185(25.1)$ & 551 (74.9) & \\
\hline Drank to get high & & & & $<0.001$ \\
\hline No & $2873(83.5)$ & $2036(70.9)$ & $837(29.1)$ & \\
\hline Yes & $568(16.5)$ & $155(27.3)$ & $413(72.7)$ & \\
\hline Problems with social media use, mean \pm SD & $2.77 \pm 1.06$ & $2.83 \pm 1.07$ & $2.68 \pm 1.03$ & $<0.001$ \\
\hline Problems with internet use, mean \pm SD & $2.57 \pm 0.88$ & $2.53 \pm 0.86$ & $2.65 \pm 0.89$ & 0.001 \\
\hline Problems with online gaming, mean \pm SD & $1.57 \pm 0.78$ & $1.56 \pm 0.76$ & $1.58 \pm 0.81$ & 0.547 \\
\hline Need to bet more money & & & & $<0.001$ \\
\hline No & $3120(92.3)$ & $2013(64.5)$ & $1107(35.5)$ & \\
\hline Yes & $261(7.7)$ & $137(52.5)$ & $124(47.5)$ & \\
\hline Lied gambling frequency & & & & $<0.001$ \\
\hline No & $3290(97.5)$ & $2107(64.0)$ & $1183(36.0)$ & \\
\hline Yes & $83(2.5)$ & $36(43.4)$ & $47(56.6)$ & \\
\hline Skipping school (days) & & & & $<0.001$ \\
\hline 0 & $2319(79.4)$ & $1580(68.1)$ & 739 (31.9) & \\
\hline $1-4$ & $503(17.2)$ & $236(46.9)$ & $267(53.1)$ & \\
\hline$\geq 5$ & $99(3.4)$ & $33(33.3)$ & $66(66.7)$ & \\
\hline Absent due to illness (days) & & & & $<0.001$ \\
\hline 0 & $1524(47.8)$ & $1041(68.3)$ & $483(31.7)$ & \\
\hline $1-4$ & $1422(44.6)$ & $850(59.8)$ & $572(40.2)$ & \\
\hline$\geq 5$ & $241(7.6)$ & $141(58.5)$ & $100(41.5)$ & \\
\hline Perceived risk in trying e-cigarettes once or twice & & & & $<0.001$ \\
\hline No & $1559(45.3)$ & $723(46.4)$ & $836(53.6)$ & \\
\hline Slight & $1263(36.7)$ & $946(74.9)$ & $317(25.1)$ & \\
\hline Moderate & $299(8.7)$ & $250(83.6)$ & $49(16.4)$ & \\
\hline Great & $149(4.3)$ & $117(78.5)$ & $32(21.5)$ & \\
\hline Don't know & $117(5.0)$ & $143(83.1)$ & $29(16.9)$ & \\
\hline Ever smoked cigarettes & & & & $<0.001$ \\
\hline Never & $2398(68.8)$ & $1980(82.7)$ & $415(17.3)$ & \\
\hline Ever & $1084(31.2)$ & $225(20.8)$ & 859 (79.2) & \\
\hline Current smoking status & & & & $<0.001$ \\
\hline Yes & 3001 (86.2) & 2147 (71.5) & $854(28.5)$ & \\
\hline No & $480(13.8)$ & $62(12.9)$ & 418 (87.1) & \\
\hline Ever alcohol use & & & & $<0.001$ \\
\hline Never & 891 (26.5) & $822(92.3)$ & $69(7.7)$ & \\
\hline Ever & $2478(73.5)$ & $1339(54.0)$ & $1139(46.0)$ & \\
\hline Current alcohol use & & & & $<0.001$ \\
\hline No & 2018 (59.3) & 1606 (79.6) & 412 (20.4) & \\
\hline Yes & $1388(40.7)$ & $569(41.0)$ & $819(59.0)$ & \\
\hline
\end{tabular}


Table 1. Continued

\begin{tabular}{|c|c|c|c|c|}
\hline Characteristics & $\begin{array}{l}\text { Total } \\
n(\%)\end{array}$ & $\begin{array}{c}\text { E-cigarette never } \\
\text { users } \\
n(\%)\end{array}$ & $\begin{array}{c}\text { E-cigarette ever } \\
\text { users } \\
n(\%)\end{array}$ & $p^{*}$ \\
\hline Current binge drinking & & & & $<0.001$ \\
\hline Never & $2326(67.1)$ & $1797(77.3)$ & $529(22.7)$ & \\
\hline Ever & 1142 (32.9) & $405(35.5)$ & $737(64.5)$ & \\
\hline Ever cannabis use & & & & $<0.001$ \\
\hline Never & $2824(81.6)$ & $2066(73.2)$ & $758(26.8)$ & \\
\hline Ever & $635(18.4)$ & $135(21.3)$ & $500(78.7)$ & \\
\hline Current cannabis use & & & & $<0.001$ \\
\hline No & 3135 (91.3) & $2152(68.6)$ & $983(31.4)$ & \\
\hline Yes & $300(8.7)$ & $44(14.7)$ & $256(85.3)$ & \\
\hline Cannabis problem use, mean \pm SD & $1.09 \pm 0.36$ & $1.01 \pm 0.16$ & $1.24 \pm 0.01$ & $<0.001$ \\
\hline Ever use of tranquilizers with prescription & & & & $<0.001$ \\
\hline Never & 3110 (89.93) & $2039(65.6)$ & $1071(34.4)$ & \\
\hline Ever & $349(10.1)$ & $155(44.1)$ & $194(55.6)$ & \\
\hline Ever use of inhalants & & & & $<0.001$ \\
\hline Never & 3106 (89.7) & $2070(66.6)$ & $1036(33.3)$ & \\
\hline Ever & $357(10.3)$ & $131(36.7)$ & $226(63.3)$ & \\
\hline \multicolumn{5}{|l|}{ Peer risk activities } \\
\hline Smoke cigarettes & $2309(69.3)$ & $1462(63.3)$ & $847(36.7)$ & 0.627 \\
\hline Drink alcoholic beverages & 2924 (87.8) & $1085(63.5)$ & $1068(36.5)$ & 0.724 \\
\hline Get drunk & 2727 (82.2) & $1728(63.4)$ & $999(36.6)$ & 0.630 \\
\hline Smoke cannabis & $1634(49.2)$ & $1035(63.3)$ & $599(36.7)$ & 0.901 \\
\hline Take tranquilizers/sedatives & $427(12.9)$ & $274(64.2)$ & $153(35.8)$ & 0.767 \\
\hline Take ecstasy & $558(16.8)$ & $350(62.7)$ & $208(37.3)$ & 0.672 \\
\hline Take inhalants & $515(15.5)$ & $320(62.1)$ & $195(37.9)$ & 0.483 \\
\hline Familial regulation & & & & 0.590 \\
\hline Know always & $2154(64.0)$ & $1368(63.5)$ & $786(36.6)$ & \\
\hline Know quite often & $794(23.6)$ & $514(64.7)$ & $280(35.3)$ & \\
\hline Know sometimes & $302(9.0)$ & $188(62.2)$ & $114(37.7)$ & \\
\hline Usually don't know & $116(3.5)$ & $68(58.6)$ & $48(41.4)$ & \\
\hline Familial support, mean \pm SD & $5.41 \pm 1.69$ & $5.40 \pm 1.70$ & $5.41 \pm 1.68$ & 0.865 \\
\hline Peer support, mean \pm SD & $5.40 \pm 1.65$ & $5.42 \pm 1.64$ & $5.36 \pm 1.66$ & 0.310 \\
\hline Relationship with mother & & & & 0.361 \\
\hline Very satisfied & $1749(52.8)$ & $1119(64.0)$ & $630(36.0)$ & \\
\hline Satisfied & 1132 (34.2) & $726(64.1)$ & $406(5.9)$ & \\
\hline Not satisfied & $433(13.1)$ & $262(60.5)$ & $171(39.5)$ & \\
\hline Relationship with father & & & & 0.318 \\
\hline Very satisfied & 1402 (43.9) & $884(63.1)$ & $518(36.9)$ & \\
\hline Satisfied & $1085(34.0)$ & 708 (65.2) & $377(34.8)$ & \\
\hline Not satisfied & 707 (22.1) & $438(61.9)$ & $269(38.0)$ & \\
\hline Smoking regulation & & & & 0.384 \\
\hline Nowhere & $1706(58.6)$ & $1077(63.1)$ & $629(36.9)$ & \\
\hline Somewhere & 1131 (38.8) & $728(64.4)$ & $403(35.6)$ & \\
\hline Anywhere & $74(2.5)$ & $42(56.8)$ & $32(43.2)$ & \\
\hline
\end{tabular}


Table 1. Continued

\begin{tabular}{l|c|c|c|} 
Characteristics & $\begin{array}{c}\text { Total } \\
n(\%)\end{array}$ & $\begin{array}{c}\text { E-cigarette never } \\
\text { users } \\
n(\%)\end{array}$ & $\begin{array}{c}\text { E-cigarette ever } \\
\text { users } \\
n(\%)\end{array}$ \\
Relationship with mother & & & \\
Very satisfied & $1749(52.8)$ & $1119(64.0)$ & $630(36.0)$ \\
Satisfied & $1132(34.2)$ & $726(64.1)$ & $406(5.9)$ \\
Not satisfied & $433(13.1)$ & $262(60.5)$ & $171(39.5)$ \\
Relationship with father & & & $518(36.9)$ \\
Very satisfied & $1402(43.9)$ & $884(63.1)$ & $377(34.8)$ \\
Satisfied & $1085(34.0)$ & $708(65.2)$ & 318 \\
Not satisfied & $707(22.1)$ & $438(61.9)$ & $269(38.0)$
\end{tabular}

*Statistical significance at $p<0.05$. a Other hobbies (play an instrument, sing, draw, write).

regulation $(\mathrm{p}<0.05)$. E-cigarette ever use was higher than cigarette ever use. More than a third (36.6\%) of the sample were e-cigarette ever users compared with $31.2 \%$ who were ever smokers and $17.3 \%(n=415)$ of those who had never tried combustible cigarettes were e-cigarette ever users. Bivariate analyses, shown in Table 2, indicate that respondents' other risk behaviors - cigarette, alcohol, cannabis, and inhalant use - have the strongest associations with e-cigarette ever use.

\section{Multivariable analysis of e-cigarette ever use}

As with the bivariate analyses, multivariable analysis (Table 2) also shows that a respondent's other risk behaviors have the strongest associations with e-cigarette ever use. Those who had ever tried cigarettes had an AOR of 4.15 (95\% CI: 1.29-13.41, $\mathrm{p}<0.05)$ for e-cigarette ever use while those who had ever used cannabis had an AOR of 2.21 (95\%
CI: $1.11-4.41, \mathrm{p}<0.05)$, and those who had ever used inhalants had an AOR of 2.51 (95\% CI: 1.07-5.88, $\mathrm{p}<0.05)$.

Compared with respondents whose parents were less well-educated, respondents with mothers who had college or university education had significantly higher odds of e-cigarette ever use $(\mathrm{AOR}=3.46$; 95\% CI: $1.40-8.54, \mathrm{p}<0.05)$. A small number of variables had significantly lower adjusted odds ratios for e-cigarette ever use, including reading books (excluding schoolbooks) for enjoyment $(\mathrm{AOR}=0.32$; 95\% CI: 0.16-0.64, p<0.05), living in a household where some rules or restrictions pertained in relation to smoking cigarettes in the house $(\mathrm{AOR}=0.53 ; 95 \%$ CI: $0.30-0.94, \mathrm{p}<0.05)$, and perceiving moderate $(\mathrm{AOR}=0.20$; 95\% CI: 0.07-0.67, $\mathrm{p}<0.05)$ risk in using e-cigarettes even once or twice. Current alcohol use was also negatively associated with e-cigarette ever use $(\mathrm{AOR}=0.44 ; 95 \%$ CI: $0.20-0.96, \mathrm{p}<0.05)$.

Table 2. Bivariate and multivariable (stepwise) logistic regression of e-cigarette ever use among people aged 15-17 years $(\mathrm{N}=3495)$

\begin{tabular}{llc}
\hline Covariates & OR $(95 \%$ CI $) *$ & AOR $(95 \%$ CI $* *$ \\
\hline Age (years) & & 1 \\
\hline 15 (Ref.) & 1 & $0.79(0.34-1.85)$ \\
16 & $1.14(0.96-1.35)$ & $0.43(0.117-1.08)$ \\
17 & $1.16(0.94-1.43)$ & 1 \\
\hline Father's education level & 1 & $2.10(0.97-4.55)$ \\
Some secondary school or completed primary school (Ref.) & $0.85(0.68-1.06)$ & -
\end{tabular}


Table 2. Continued

\begin{tabular}{|c|c|c|}
\hline Covariates & OR $(95 \% \mathrm{CI}) *$ & $\operatorname{AOR}(95 \% \mathrm{CI}) *$ \\
\hline \multicolumn{3}{|l|}{ Mother's education level } \\
\hline Some secondary school or completed primary school (Ref.) & 1 & 1 \\
\hline Completed secondary school & $0.78(0.59-1.03)$ & $3.46(1.40-8.54)$ \\
\hline College or university & $1.35(1.06-1.71)$ & - \\
\hline \multicolumn{3}{|l|}{ Perceived wealth } \\
\hline About the same (Ref.) & 1 & 1 \\
\hline Much better off & $1.14(0.92-1.40)$ & $0.48(0.59-1.47)$ \\
\hline Better off & $1.01(0.86-1.19)$ & $0.49(0.24-1.02)$ \\
\hline Less well off & $1.26(0.98-1.61)$ & - \\
\hline \multicolumn{3}{|l|}{ Household composition } \\
\hline Two parents (Ref.) & 1 & 1 \\
\hline One parent & $1.12(0.92-1.35)$ & $1.34(0.34-5.32)$ \\
\hline Blended families & $1.07(0.80-1.46)$ & $0.72(0.17-3.11)$ \\
\hline \multicolumn{3}{|l|}{ Average grade } \\
\hline$A$ and $B$ (Ref.) & 1 & 1 \\
\hline C & $1.15(0.99-1.34)$ & $1.19(0.66-2.16)$ \\
\hline D or lower & $1.14(0.91-1.44)$ & $1.40(0.53-3.74)$ \\
\hline Read books, no vs yes & $0.43(0.36-0.51)$ & $0.32(0.16-0.64)$ \\
\hline Actively participate in sports, no vs yes & $1.01(0.84-1.22)$ & $1.17(0.57-2.41)$ \\
\hline Other hobbies, no vs yes & $0.76(0.66-0.87)$ & $1.38(0.77-2.46)$ \\
\hline \multicolumn{3}{|l|}{ Age of smoking initiation (years) } \\
\hline$<13$ (Ref.) & 1 & 1 \\
\hline$\geq 14$ & $3.49(3.03-4.02)$ & $0.70(0.36-1.35)$ \\
\hline \multicolumn{3}{|l|}{ Skipping school (days) } \\
\hline 0 (Ref.) & 1 & 1 \\
\hline $1-4$ & $2.42(1.99-2.94)$ & $1.19(0.62-2.89)$ \\
\hline$\geq 5$ & $4.27(2.79-6.55)$ & $0.57(0.17-1.83)$ \\
\hline \multicolumn{3}{|l|}{ Absent due to illness (days) } \\
\hline 0 (Ref.) & 1 & 1 \\
\hline $1-4$ & $1.45(1.25-1.69)$ & $1.17(0.66-2.07)$ \\
\hline$\geq 5$ & $1.53(1.16-2.02)$ & $0.23(0.09-0.61)$ \\
\hline \multicolumn{3}{|l|}{ Perceived risk in trying e-cigarettes once or twice } \\
\hline No (Ref.) & 1 & 1 \\
\hline Slight & $0.29(0.25-0.34)$ & $0.86(0.46-1.58)$ \\
\hline Moderate & $0.17(1.22-0.23)$ & $0.20(0.07-0.67)$ \\
\hline Great & $0.24(0.16-0.35)$ & $0.42(0.07-2.56)$ \\
\hline Don't know & $0.17(0.12-0.26)$ & - \\
\hline Ever use of cigarettes, never vs ever & $18.21(15.2-21.83)$ & $4.15(1.29-13.41)$ \\
\hline Current cigarette use, no vs yes & $16.90(12.83-22.39)$ & $1.64(0.85-3.16)$ \\
\hline Ever alcohol use, never vs ever & $10.13(7.83-13.11)$ & $2.38(0.31-18.52)$ \\
\hline Current alcohol use, no vs yes & $5.61(4.82-6.53)$ & $0.44(0.20-0.96)$ \\
\hline Current binge drinking, no vs yes & $6.18(5.29-7.22)$ & $1.80(0.91-3.55)$ \\
\hline Cannabis ever use, no vs yes & $10.09(8.20-12.42)$ & $2.21(1.11-4.41)$ \\
\hline
\end{tabular}


Table 2. Continued

\begin{tabular}{|c|c|c|}
\hline Covariates & OR $(95 \% \mathrm{CI}) *$ & $\operatorname{AOR}(95 \% \mathrm{CI}) *$ \\
\hline Cannabis problem use & $16.79(10.75-26.23)$ & $2.78(0.97-7.99)$ \\
\hline Ever use of inhalants, never vs ever & $3.45(2.74-4.33)$ & $2.51(1.07-5.88)$ \\
\hline \multicolumn{3}{|l|}{ Peer risk activities, no vs yes } \\
\hline Get drunk & $1.05(0.87-1.26)$ & $1.32(0.61-2.86)$ \\
\hline Smoke cannabis & $1.01(0.88-1.16)$ & $1.80(0.93-3.47)$ \\
\hline Take tranquilizers/sedatives & $0.97(0.78-1.20)$ & $0.45(0.15-1.35)$ \\
\hline Take inhalants & $1.07(0.88-1.30)$ & $1.54(0.52-4.62)$ \\
\hline \multicolumn{3}{|l|}{ Familial regulation } \\
\hline Know always (Ref.) & 1 & 1 \\
\hline Know quite often & $0.95(0.80-1.12)$ & $0.52(0.26-1.04)$ \\
\hline Know sometimes & $1.05(0.82-1.35)$ & $1.68(0.45-6.22)$ \\
\hline Usually don't know & $1.23(0.84-1.80)$ & - \\
\hline Peer support & $0.98(0.94-1.02)$ & $1.06(0.88-1.27)$ \\
\hline \multicolumn{3}{|l|}{ Relationship with mother } \\
\hline Very satisfied (Ref.) & 1 & 1 \\
\hline Satisfied & $0.99(0.85-1.16)$ & $1.45(0.66-3.16)$ \\
\hline Not satisfied & $1.16(0.93-1.44)$ & $1.87(0.63-5.52)$ \\
\hline \multicolumn{3}{|l|}{ Relationship with father } \\
\hline Very satisfied (Ref.) & 1 & 1 \\
\hline Satisfied & $0.91(0.77-1.07)$ & - \\
\hline Not satisfied & $1.05(0.87-1.26)$ & $0.50(0.20-1.22)$ \\
\hline \multicolumn{3}{|l|}{ Smoking regulation } \\
\hline Nowhere (Ref.) & 1 & 1 \\
\hline Somewhere & $0.94(0.81-1.11)$ & $0.53(0.30-0.94)$ \\
\hline Anywhere & $1.30(0.81-2.09)$ & $0.54(0.05-7.04)$ \\
\hline
\end{tabular}

AOR: adjusted odds ratio. *Bold indicates statistical significance at $p<0.05$.

\section{DISCUSSION}

\section{Sociodemographic influences: social class, sex, and household composition}

To date, findings about e-cigarette use and social class have been ambivalent. Recent Irish research found that, in a sample in which smoking was patterned by social class, e-cigarette ever use was not ${ }^{23}$. This study found that perceived relative wealth was not statistically significantly associated with e-cigarette ever use but that parental education level was. This suggests some differences in teenagers' views and motivations regarding e-cigarettes compared with cigarettes. The association between smoking and lower socioeconomic status is well-established, but the association with other substances is more ambivalent. For example, young adults with the highest family background SES have been found to be most prone to alcohol and marijuana use, even after adjusting for covariates ${ }^{24}$. They have also been found to be more likely to use other drugs, and to use alcohol and other substances to cope with stress ${ }^{25}$. Our findings about increased e-cigarette ever use among people aged 1517 years with higher-educated mothers $(\mathrm{AOR}=3.46$; 95\% CI: 1.40-8.54) may indicate that e-cigarette ever use has more in common with alcohol and other drug use than it has with smoking or that more-educated parents have different attitudes to e-cigarettes than less-educated parents.

Other familial behaviors and supports were also implicated in adolescent e-cigarette ever use. Even after adjusting for covariates, living in a household where some rules or restrictions were in place 
regarding whether or where people could smoke in the house lowered the odds of people aged $15-17$ years e-cigarette ever use (AOR=0.53; 95\% CI: 0.30-0.94)

Parental anti-smoking communication and encouragement reduces teenage smoking ${ }^{26}$. Our findings extend the role of parental influence, including having no-smoking rules in the home, from cigarette smoking to e-cigarette ever use, suggesting an encouraging role for parents in reducing nicotine consumption in teenagers.

\section{Personal behaviors}

Polysubstance use is highly prevalent among adolescents who use e-cigarettes ${ }^{27}$. E-cigarette ever use was strongly associated with ever use of tobacco, cannabis, and inhalants, and the association was especially strong for cigarette smoking. Risk-taking, indicated by experimenting with many substances, may be implicated in e-cigarette ever use and these findings provide some support for the common liability theory ${ }^{17}$. We agree ${ }^{28}$ that e-cigarette screening should include the assessment of other substances, especially cigarettes, alcohol, and cannabis, with a view to identifying and implementing prevention efforts and improving population health.

When we adjusted for covariates in our regression model, reading books for enjoyment remained protective against e-cigarette ever use $(\mathrm{AOR}=0.32$; 95\% CI: 0.36-0.51). Perceiving moderate risk in using e-cigarettes is indicated as protective against e-cigarette ever use (AOR=0.20; 95\% CI: 0.07-0.67), suggesting a role for health education in providing clear, focused, up-to-date information for adolescents about the risks of e-cigarette ever use. Efforts should be stepped up in the junior cycle of post-primary schooling to develop health education curricula that are appropriate in terms of content, pedagogy, resources and evaluation ${ }^{29}$.

\section{Peer influences}

Studies have shown that adolescents are more likely to engage in risky behaviors in the presence of peers ${ }^{30,31}$. In our model, we tested correlations between all peer substance use [use of tobacco, alcohol (including getting drunk), cannabis, tranquilizers/sedatives, ecstasy, inhalants] and e-cigarette ever use and increased odds ratios were noted but data were not sufficiently sensitive to detect differences.

\section{Experimentation, continuation, and cessation}

E-cigarette ever use is largely associated with teenagers' other substance use. Experimentation is widely recognized as a feature of adolescence and others have drawn 'a positive, linear relation between substance use and psychopathology, such that the more frequently children and adolescents use illegal substances, the greater their risk for exhibiting internalizing or externalizing psychiatric disorders ${ }^{32}$, suggesting that complete abstinence among children and adolescence is seen as a desirable outcome. At a minimum, an increased proportion of those who ever use e-cigarettes experimentally will go on to become addicted users of nicotine ${ }^{33}$, or possibly dual users of e-cigarettes and combustible tobacco ${ }^{34,35}$. Marijuana use has also been found to increase at a faster rate among e-cigarette users when compared to their peers who used cigarettes or a combination of cigarettes and e-cigarettes (dual users) ${ }^{36}$. Unlike tobacco, e-cigarettes in Ireland are currently largely unregulated. The findings suggest a role for health education highlighting the important link in e-cigarette ever use with peer and personal polysubstance use.

\section{Limitations}

The study captures the associations of ever use of e-cigarettes at a point in time of school-going people aged 15-17 years in Ireland. It cannot tell us about the small percentage of students who do not attend school in Ireland at that age and may be different. ESPAD questionnaires are completed by students in a school setting therefore self-reporting bias is a consideration. Social desirability bias is a further consideration given the sensitive nature of the behavior under study - teenage substance use. Also, e-cigarette use is changing rapidly so advice on regulation and control needs regular up-dating. The use of the stepwise regression method produces confidence intervals around the parameter estimates that are too narrow and $\mathrm{p}$-values that are too low due to multiple comparisons. Longitudinal and qualitative studies are certainly needed to improve our understanding and predictions for the future, but these exploratory analyses give us valuable insights in the present situation.

\section{CONCLUSIONS}

E-cigarette ever users are more likely to be male and to have higher-educated mothers. While sex and 
parental education level are associated with e-cigarette ever use, our multivariable analyses show that these influences wane in comparison with teenagers' personal risk behaviors, particularly in terms of their polysubstance use, but cigarette use is the most strongly associated with e-cigarette ever use with odds greater than 4 times. Our findings emphasize the importance and usefulness of regulation of cigarette smoking in the home in preventing ever use of e-cigarettes. The perception of risk of e-cigarette use is also shown to be associated with ever use and this also may be influenced by e-cigarette regulation in the home. Education at school about e-cigarette use is largely absent or inadequate in many European countries, including Ireland, and needs strengthening ${ }^{29}$. Parents are important modifiers of adolescents' nicotine use and we recommend that school-based education be extended to include interventions aimed at parents. Parental attitudes on the dangers of teenagers' e-cigarette ever use are not well-known and need further study.

\section{REFERENCES}

1. Sunday S, Keogan S, Hanafin J, Clancy L. ESPAD 2019 Ireland: Results from the European Schools Project on Alcohol and Other Drugs in Ireland. TobaccoFree Research Institute Ireland; 2020. Accessed November 24, 2021. https://www.drugsandalcohol.ie/33347/1/ ESPAD\%202019\%20Ireland.pdf

2. ESPAD Group. ESPAD Report 2019: Results from the European School Survey Project on Alcohol and Other Drugs. European Monitoring Centre for Drugs and Drug Addiction Joint Publications, European School Survey Project on Alcohol and Other Drugs, Publications Office of the European Union; 2020. doi:10.2810/877033

3. Evans-Polce R, Veliz P, Boyd CJ, McCabe VV, McCabe SE. Trends in E-Cigarette, Cigarette, Cigar, and Smokeless Tobacco Use Among US Adolescent Cohorts, 20142018. Am J Public Health. 2020;110(2):163-165. doi:10.2105/AJPH.2019.305421

4. Gentzke AS, Creamer M, Cullen KA, et al. Vital Signs: Tobacco Product Use Among Middle and High School Students - United States, 2011-2018. MMWR Morb Mortal Wkly Rep. 2019;68(6):157. doi:10.15585/mmwr.mm6806e1

5. Wipfli H, Bhuiyan MR, Qin X, et al. Tobacco use and E-cigarette regulation: Perspectives of University Students in the Asia-Pacific. Addict Behav. 2020;107:106420. doi:10.1016/j.addbeh.2020.106420

6. Hanafin J, Sunday S, Clancy L. E-cigarettes and smoking in Irish teens: a logistic regression analysis of current (past 30-day) use of e-cigarettes. Journal of Public
Health. 2021;1-12. doi:10.1007/s10389-021-01610-1

7. Sunday S, Hanafin J, Clancy L. Increased smoking and e-cigarette use among Irish teenagers: a new threat to Tobacco Free Ireland 2025. ERJ Open Res. 2021;7(4):004382021. doi:10.1183/23120541.00438-2021

8. Scottish Government. Scottish Schools Adolescent Lifestyle and Substance Use Survey (SALSUS): Smoking Report (2018). Accessed July 9, 2021. https://www.gov. scot/publications/scottish-schools-adolescent-lifestylesubstance-use-survey-salsus-smoking-report-2018/

9. NHS Digital. Smoking, Drinking and Drug Use among Young People in England 2018 [NS]. NHS Digital; 2019. Accessed October 11, 2021. https://digital.nhs. uk/data-and-information/publications/statistical/ smoking-drinking-and-drug-use-among-young-peoplein-england/2018

10. Cullen KA, Gentzke AS, Sawdey MD, et al. e-Cigarette Use Among Youth in the United States, 2019. JAMA. 2019;322(21):2095-2103. doi:10.1001/jama.2019.18387

11. Kapan A, Stefanac S, Sandner I, Haider S, Grabovac I, Dorner TE. Use of Electronic Cigarettes in European Populations: A Narrative Review. Int J Environ Res Public Health. 2020;17(6):1971. doi:10.3390/ijerph17061971

12. Porcellato L, Ross-Houle K, Quigg Z, et al. Welsh Primary Schoolchildren's Perceptions of Electronic Cigarettes: A Mixed Methods Study. Int J Environ Res Public Health. 2020;17(10):3639. doi:10.3390/ijerph17103639

13. Tokle R. 'Vaping and fidget-spinners': A qualitative, longitudinal study of e-cigarettes in adolescence. Int J Drug Policy. 2020;82:102791. doi:10.1016/j.drugpo.2020.102791

14. Chaffee BW, Watkins SL, Glantz SA. Electronic Cigarette Use and Progression From Experimentation to Established Smoking. Pediatrics. 2018;141(4):e20173594. doi:10.1542/peds.2017-3594

15. Li S, Levy D, Clancy L. Tobacco Free Ireland 2025: SimSmoke prediction for the end game. Tob Prev Cessat. 2018;4(June). doi:10.18332/tpc/91427

16. Kandel ER, Kandel DB. A Molecular Basis for Nicotine as a Gateway Drug. N Engl J Med. 2014;371(10):932-943. doi:10.1056/NEJMsa1405092

17. Vanyukov MM, Tarter RE, Kirillova GP, et al. Common liability to addiction and "gateway hypothesis": theoretical, empirical and evolutionary perspective. Drug Alcohol Depend. 2012;123 Suppl 1(Suppl 1):S3-S17. doi:10.1016/j.drugalcdep.2011.12.018

18. Schneider S, Diehl K. Vaping as a Catalyst for Smoking? An Initial Model on the Initiation of Electronic Cigarette Use and the Transition to Tobacco Smoking Among Adolescents. Nicotine Tob Res. 2016;18(5):647-653. doi:10.1093/ntr/ntv193

19. Selya AS, Foxon F. Trends in electronic cigarette use and conventional smoking: quantifying a possible 'diversion' effect among US adolescents. Addiction. 2021;116(7):1848-1858. doi:10.1111/add.15385

20. Cheng HG, Largo EG, Gogova M. E-cigarette use and 
onset of first cigarette smoking among adolescents: An empirical test of the 'common liability' theory. F1000Res. 2019;8:2099. doi:10.12688/f1000research.21377.3

21. Fadus MC, Smith TT, Squeglia LM. The rise of e-cigarettes, pod mod devices, and JUUL among youth: Factors influencing use, health implications, and downstream effects. Drug Alcohol Depend. 2019;201:8593. doi:10.1016/j.drugalcdep.2019.04.011

22. Hanafin J, Sunday S, Clancy L. Friends and Family Matter Most: a trend analysis of increasing e-cigarette use among Irish teenagers and socio-demographic, personal, peer and familial associations. BMC Public Health. 2021;21:112. doi:10.1186/s12889-021-12113-9

23. Költő A, Gavin A, Molcho M, Kelly C, Walker L, Nic Gabhainn S. The Irish Health Behaviour in Schoolaged Children (HBSC) Study 2018. Department of Health and National University of Ireland; 2020. doi:10.13025/zvwm-rp65

24. Patrick ME, Wightman P, Schoeni RF, Schulenberg JE. Socioeconomic status and substance use among young adults: a comparison across constructs and drugs. J Stud Alcohol Drugs. 2012;73(5):772-782. doi:10.15288/jsad.2012.73.772

25. Martin CC. High Socioeconomic Status Predicts Substance Use and Alcohol Consumption in U.S. Undergraduates. Subst Use Misuse. 2019;54(6):1035-1043. doi:10.1080/10826084.2018.1559193

26. Broun A, Haynie D, Choi K. Parental anti-smoking encouragement as a longitudinal predictor of young adult cigarette and e-cigarette use in a US national study. Nicotine Tob Res. 2021;23(9):1468-1474. doi:10.1093/ntr/ntab026

27. Henriksen L, Jackson C. Anti-smoking socialization: relationship to parent and child smoking status. Health Commun. 1998;10(1):87-101. doi:10.1207/s15327027hc1001_5

28. Gilbert PA, Kava CM, Afifi R. High-School Students Rarely Use E-Cigarettes Alone: A Sociodemographic Analysis of Polysubstance Use Among Adolescents in the United States. Nicotine Tob Res. 2021;23(3):505-510. doi:10.1093/ntr/ntaa037

29. Hanafin J, Clancy L. Youth Smoking in Europe: Strategies for Prevention and Reduction. TobaccoFree Research Institute Ireland; 2019. Accessed November 24, 2021. https://www.tri.ie/publications.html

30. Gardner M, Steinberg L. Peer influence on risk taking, risk preference, and risky decision making in adolescence and adulthood: an experimental study. Dev Psychol. 2005;41(4):625. doi:10.1037/0012-1649.41.4.625

31. Chein J, Albert D, O'Brien L, Uckert K, Steinberg L. Peers increase adolescent risk taking by enhancing activity in the brain's reward circuitry. Dev Sci. 2011;14(2):F1-F10. doi:10.1111/j.1467-7687.2010.01035.x

32. Milich R, Lynam D, Zimmerman R, et al. Differences in young adult psychopathology among drug abstainers, experimenters, and frequent users. J Subst Abuse. 2000;11(1):69-88. doi:10.1016/s0899-3289(99)00021-8

33. Klein JD. E-Cigarettes: A 1-Way Street to Traditional Smoking and Nicotine Addiction for Youth. Pediatrics. 2018;141(1):e20172850. doi:10.1542/peds.2017-2850

34. Jeon C, Jung KJ, Kimm H, et al. E-cigarettes, conventional cigarettes, and dual use in Korean adolescents and university students: Prevalence and risk factors. Drug Alcohol Depend. 2016;168:99-103. doi:10.1016/j.drugalcdep.2016.08.636

35. Smith DM, Gawron M, Balwicki L, Sobczak A, Matynia M, Goniewicz ML. Exclusive versus dual use of tobacco and electronic cigarettes among adolescents in Poland, 2010-2016. Addict Behav. 2019;90:341-348. doi:10.1016/j.addbeh.2018.11.035

36. Veliz PT, McCabe SE, Evans-Polce RJ, Boyd CJ. Assessing how the history of e-cigarette and cigarette use are associated with the developmental course of marijuana use in a sample of United States adolescents. Drug Alcohol Depend. 2020;216:108308. doi:10.1016/j.drugalcdep.2020.108308

\section{ACKNOWLEDGEMENTS}

We thank the RCDHTrust and the Department of Health Ireland for their financial support We also acknowledge the important part played by colleagues, Sheila Keogan, in the management and supervision of data collection for ESPAD Ireland 2019, and Ermelinda Furtado da Luz Brzychcyk in data collection.

\section{CONFLICTS OF INTEREST}

The authors have completed and submitted the ICMJE Form for Disclosure of Potential Conflicts of Interest and none was reported.

\section{FUNDING}

Payments were made to the institution (TFRI) from the Royal City of Dublin Hospital Dublin Trust, Department of Health Ireland (RCDHT Grant No. 184, ESPAD 2019 Contract). The funders had no role in the study design; in the collection, analysis and interpretation of data; in the writing of the articles; and in the decision to submit it for publication.

\section{ETHICAL APPROVAL AND INFORMED CONSENT}

Ethical approval (REC2018-126; 29 January 2019) was obtained from the Research and Ethics Committee of Technological University Dublin. Informed consent was not required as data were from an existing survey database.

\section{DATA AVAILABILITY}

The data supporting this research are available from the authors on reasonable request.

\section{AUTHORS' CONTRIBUTIONS}

$\mathrm{JH}$ : conceptualization, methodology, writing of original draft, reviewing and editing, and supervision. SS: formal analysis, data curation, visualization, statistical examination, reviewing and editing. LC: conceptualization, supervision visualization, investigation, reviewing and editing, resources, and funding acquisition.

\section{PROVENANCE AND PEER REVIEW}

Not commissioned; externally peer reviewed. 